\section{S sciendo}

DOI: $10.2478 /$ awutm-2018-0012
Analele Universităţii de Vest,

Timişoara

Seria Matematică - Informatică

LVI, 2, (2018), 3- 12

\title{
Stability of Picard operators under operator perturbations
}

Adrian Petruşel and Ioan A. Rus

Dedicated to Professor Mihail Megan on the occasion of his 70th birthday

\begin{abstract}
In this paper we study the following problems:
I. Let $(M, d)$ be a complete metric space and $f, g: M \rightarrow M$ be two operators. We suppose that:

(a) $f$ is a Picard operator with its unique fixed point $x_{f}^{*}$;

(b) there exists $\eta>0$ such that $d(f(x), g(x)) \leq \eta$, for every $x \in M$.

The problem consists in estimating $d\left(g^{n}(x), x_{f}^{*}\right)$, for $x \in M$ and $n \in \mathbb{N}^{*}$.

II. Let $B$ be a Banach space and $f, g: B \rightarrow B$ be two operators. We suppose that $f$ is a Picard operator. The problem is to find sufficient conditions which guarantee that $f+g$ is a Picard operator.
\end{abstract}

AMS Subject Classification (2000). 47H10; 47H09; 54H25; 34D10; 45M10; 54H25

Keywords. metric spaces; fixed point; Picard operator; operator perturbation; data dependence of the fixed point.

\section{Introduction}

There exist various aspects of the stability problem for the solutions of differential and integral equations (see [1], [5], [18], [21], [26], [31], [37], ...), of operatorial equations ([7], [2], [4], [6], [8], [12], [17], [18], [23], [25], [27], [28], 
[29], [30], [33], [34], [36], ...) and of functional-operatorial equations ([3], [10], [11], [14], [15], [16], [20], [22], [24], [32], [33], ...).

In this paper, we will study the following two abstract problems:

Problem I. (see Problem 1.4.2 in [30]) Let $(M, d)$ be a complete metric space and $f, g: M \rightarrow M$ be two operators. We suppose that:

(a) $f$ is a Picard operator with its unique fixed point $x_{f}^{*}$;

(b) there exists $\eta>0$ such that $d(f(x), g(x)) \leq \eta$, for every $x \in M$.

The problem consists in estimating $d\left(g^{n}(x), x_{f}^{*}\right)$, for $x \in M$ and $n \in \mathbb{N}^{*}$.

Problem II. (see Problem 55 in [34]) Let $B$ be a Banach space and $f, g$ : $B \rightarrow B$ be two operators. We suppose that $f$ is a Picard operator. The problem is to find sufficient conditions which guarantee that $f+g$ is a Picard operator.

We will present first some basic notions and results which are essential for the main part of this paper.

Let $X$ be a nonempty set and $f: X \rightarrow X$ be an operator. We consider the fixed point equation

$$
x=f(x), x \in X .
$$

We denote by $F_{f}$ the fixed point set of $f$, i.e., $F_{f}:=\{x \in X \mid f(x)=x\}$. If $(M, d)$ is a metric space, then, by definition, $f$ is a Picard operator if $F_{f}=\left\{x^{*}\right\}$ and

$$
f^{n}(x) \rightarrow x^{*} \text { as } n \rightarrow \infty \text {, for all } x \in M \text {. }
$$

A Picard operator $f$ for which there exists a function $\psi: \mathbb{R}_{+} \rightarrow \mathbb{R}_{+}$increasing, continuous in 0 and satisfying $\psi(0)=0$, such that

$$
d\left(x, x^{*}\right) \leq \psi(d(x, f(x)), \text { for all } x \in X,
$$

is called a $\psi$-Picard operator. In particular, if $\psi$ has a linear form (i.e., $\psi(t)=c t, t \in \mathbb{R}_{+}$, for some $c>0$ ), then $f$ is called a $c$-Picard operator.

\section{Definition 1.1.}

Let $(M, d)$ be a metric space and $f: M \rightarrow M$ be an operator. Then, $f$ is called:

(i) an $\alpha$-contraction if $\alpha \in] 0,1[$ and

$$
d(f(x), f(y)) \leq \alpha d(x, y), \text { for every } x, y \in M \text {. }
$$

(ii) a Kannan operator if there exists $\beta \in] 0, \frac{1}{2}[$ such that

$$
d(f(x), f(y)) \leq \beta(d(x, f(x))+d(y, f(y))), \text { for every } x, y \in M .
$$


(iii) a $\varphi$-contraction if $\varphi: \mathbb{R}_{+} \rightarrow \mathbb{R}_{+}$is a comparison function (i.e., $\varphi$ is increasing and $\varphi^{n}(t) \rightarrow 0$ as $n \rightarrow \infty$, for every $t \in \mathbb{R}_{+}$) and

$$
d(f(x), f(y)) \leq \varphi(d(x, y)), \text { for every } x, y \in M .
$$

(iv) a Hardy-Rogers operator if there are $\alpha, \beta, \gamma \in \mathbb{R}_{+}$with $\alpha+2 \beta+2 \gamma<1$, such that, for every $x, y \in M$, we have

$$
\begin{gathered}
d(f(x), f(y)) \leq \\
\alpha d(x, y)+\beta(d(x, f(x))+d(y, f(y))) \\
+\gamma(d(x, f(y))+d(y, f(x))) .
\end{gathered}
$$

\section{Example 1.1.}

1) If $(M, d)$ is a complete metric space and $f: M \rightarrow M$ is an $\alpha$-contraction, then $f$ is a $\frac{1}{1-\alpha}$-Picard operator.

2 ) If $(M, d)$ is a complete metric space and $f: M \rightarrow M$ is Kannan operator with constant $\beta$, then $f$ is a $\frac{1-\beta}{1-2 \beta}$-Picard operator.

3) If $(M, d)$ is a complete metric space and $f: M \rightarrow M$ is Hardy-Rogers operator with constants $\alpha, \beta, \gamma$, then $f$ is a $\frac{1-\beta-\gamma}{1-\alpha-2 \beta-2 \gamma}$-Picard operator.

4) If $(M, d)$ is a complete metric space and $f: M \rightarrow M$ is $\varphi$-contraction, such that $\varphi$ is a strict comparison function (i.e., $\varphi$ is a comparison function and $t-\varphi(t) \rightarrow \infty$ as $t \rightarrow \infty$, then the operator $f$ is a $\psi_{\varphi}$-Picard operator, with $\psi_{\varphi}(t):=\sup \left\{s \in \mathbb{R}_{+}: s-\varphi(s) \leq t\right\}$.

Other examples of Picard operator involving function spaces are given now.

\section{Example 1.2.}

Let $(B,|\cdot|)$ be a Banach space and $g \in C([a, b] \times B, B)$ be an operator. We suppose that there exists $\left.l_{g} \in\right] 0,1[$ such that

$$
\left|g(t, u)-g(t, v) \leq l_{g}\right| u-v \mid, \forall t \in[a, b], \forall u, v \in B .
$$

Consider $T: C([a, b], B) \rightarrow C([a, b], B)$ defined by

$$
T x(t):=g(t, x(t)), t \in[a, b] .
$$

If we consider the Banach space $\left.\mathbb{B}:=(C([a, b], B),\|\cdot\|)_{\infty}\right)$, then the operator $T: \mathbb{B} \rightarrow \mathbb{B}$ is an $l_{g}$-contraction and, hence, a Picard operator.

\section{Example 1.3.}

Let $(B,|\cdot|)$ be a Banach space and $K \in C([a, b] \times[a, b] \times B, B)$ be an operator. We suppose that there exists $\left.l_{K} \in\right] 0,1[$ such that

$$
\left|K(t, s, u)-K(t, s, v) \leq l_{K}\right| u-v \mid, \forall t, s \in[a, b], \forall u, v \in B
$$


Consider $S: C([a, b], B) \rightarrow C([a, b], B)$ defined by

$$
S x(t):=\int_{a}^{t} K(t, s, x(s)) d s, t \in[a, b] .
$$

If we consider the Banach space $\left.\mathbb{B}:=(C([a, b], B),\|\cdot\|)_{\tau}\right)$ (where $\|\cdot\|_{\tau}$ is a Bielecki type norm with $\tau>l_{K}$ ), then the operator $S: \mathbb{B} \rightarrow \mathbb{B}$ is $\frac{l_{K}}{\tau}$-contraction and, hence, a Picard operator. Moreover, $S$ is a strong contraction in the sense of Goebel. Recall that if $(B,\|\cdot\|)$ is a Banach space, then an operator $f: B \rightarrow B$ is called a strong contraction if for every $\epsilon>0$ there exists a norm $\|\cdot\|_{\epsilon}$ on $B$, equivalent with $\|\cdot\|$, such that

$$
\|f(x)-f(y)\|_{\epsilon} \leq \epsilon\|x-y\|_{\epsilon} \text {, for every } x, y \in B .
$$

For details see [13] and [34].

\section{Results concerning Problem I.}

For a better understanding of the problem, we present several examples.

\section{Example 2.1.}

If $(M, d)$ is a complete metric space and $f: M \rightarrow M$ is an $\alpha$-contraction, then, for Problem I, we have the following result.

Theorem 2.1. Let $(M, d)$ is a complete metric space, $f: M \rightarrow M$ be an $\alpha$-contraction and $g: M \rightarrow M$ be an operator for which there exists $\eta>0$ such that $d(f(x), g(x)) \leq \eta$, for every $x \in M$. Denote by $x_{f}^{*}$ the unique fixed point of $f$. Then

$$
d\left(g^{n}(x), x_{f}^{*}\right) \leq \frac{\eta}{1-\alpha}+\frac{\alpha^{n}}{1-\alpha} d(x, f(x)), \forall x \in M, \forall n \in \mathbb{N}^{*} .
$$

Proof. For $x \in M$, we have that

$$
\begin{gathered}
d\left(g^{n}(x), x_{f}^{*}\right) \leq d\left(f^{n}(x), x_{f}^{*}\right)+d\left(f^{n}(x), g^{n}(x)\right) \leq \\
\frac{\alpha^{n}}{1-\alpha} d(x, f(x))+d\left(f^{n}(x), g^{n}(x)\right) .
\end{gathered}
$$

On the other hand

$$
\begin{aligned}
& d\left(f^{n}(x), g^{n}(x)\right) \leq d\left(f\left(g^{n-1}(x)\right), g\left(g^{n-1}(x)\right)\right)+d\left(f\left(g^{n-1}(x)\right), f\left(f^{n-1}(x)\right)\right) \leq \\
& \eta+\alpha d\left(f^{n-1}(x), g^{n-1}(x)\right) \leq \eta+\alpha \eta+\alpha^{2} d\left(f^{n-2}(x), g^{n-2}(x)\right) \leq \cdots \leq \frac{\eta}{1-\alpha} .
\end{aligned}
$$

Thus, the conclusion follows by the above two relations. 


\section{Example 2.2.}

Let $(M, d)$ be a complete metric space and $f: M \rightarrow M$ be a $\varphi$-contraction. Suppose that, in addition, $\varphi$ satisfies the following assumptions:

(a) $t-\varphi(t) \rightarrow \infty$, as $t \rightarrow \infty$ (i.e., $\varphi$ is a strict comparison function);

(b) $\varphi\left(t_{1}+t_{2}\right) \leq \varphi\left(t_{1}\right)+\varphi\left(t_{2}\right), \forall t_{1}, t_{2} \in \mathbb{R}_{+}$.

Then, for Problem I, we have the following result.

Theorem 2.2. Let $(M, d)$ be a complete metric space and $f: M \rightarrow M$ be a strong $\varphi$-contraction satisfying (a)-(b) from above. Denote by $x_{f}^{*}$ the unique fixed point of $f$. If $g: M \rightarrow M$ is an operator for which there exists $\eta>0$ such that $d(f(x), g(x)) \leq \eta$ for every $x \in M$, then we have that

$$
d\left(g^{n}(x), x_{f}^{*}\right) \leq \theta(\eta)+\varphi^{n}\left(\psi_{\varphi}(d(x, f(x)))\right), \forall x \in M, \forall n \in \mathbb{N}^{*},
$$

where $\theta(t):=\sum_{n=0}^{\infty} \varphi^{n}(t)$ and $\psi_{\varphi}(t)=\sup \left\{s \in \mathbb{R}_{+}: s-\varphi(s) \leq t\right\}$, for $t \in \mathbb{R}_{+}$.

Proof. If $\varphi$ is a strict comparison function, then the function $\psi_{\varphi}: \mathbb{R}_{+} \rightarrow \mathbb{R}_{+}$ defined by $\psi_{\varphi}(t)=\sup \left\{s \in \mathbb{R}_{+}: s-\varphi(s) \leq t\right\}$ is increasing and has the property that $\psi_{\varphi}(t) \rightarrow 0_{+}$as $t \rightarrow 0_{+}$. Then, for each $x \in M$, we have

$$
d\left(g^{n}(x), x_{f}^{*}\right) \leq d\left(f^{n}(x), x_{f}^{*}\right)+d\left(f^{n}(x), g^{n}(x)\right) .
$$

On one hand

$$
d\left(f^{n}(x), x_{f}^{*}\right) \leq \varphi^{n}\left(d\left(x, x_{f}^{*}\right)\right)
$$

with

$$
d\left(x, x_{f}^{*}\right) \leq \psi_{\varphi}(d(x, f(x)))
$$

On the other hand

$$
\begin{gathered}
d\left(f^{n}(x), g^{n}(x)\right) \leq d\left(f\left(g^{n-1}(x)\right), g\left(g^{n-1}(x)\right)\right)+d\left(f\left(g^{n-1}(x)\right), f\left(f^{n-1}(x)\right)\right) \leq \\
\eta+\varphi\left(d\left(f^{n-1}(x), g^{n-1}(x)\right)\right) \leq \eta+\varphi\left(\eta+\varphi\left(d\left(f^{n-2}(x), g^{n-2}(x)\right)\right)\right) \leq \\
\eta+\varphi(\eta)+\varphi^{2}\left(d\left(f^{n-2}(x), g^{n-2}(x)\right)\right) \leq \cdots \leq \sum_{n=0}^{\infty} \varphi^{n}(\eta)=\theta(\eta) .
\end{gathered}
$$

Thus, the conclusion follows by the above two relations.

We formulate now two open problems.

Problem A. For which generalized contractions (Kannan operators, HardyRogers operators, ...) in complete metric spaces we have similar results to the above ones? 
Problem B. A second open question is to study the above problem in the case of generalized metric spaces.

Concerning the last problem, we have the following result in complete $\mathbb{R}_{+}^{m}$ metric spaces.

\section{Example 2.3.}

If $(M, d)$ is a complete $\mathbb{R}_{+}^{m}$-metric space and $f: M \rightarrow M$ is an $A$-contraction (i.e., $A \in \mathcal{M}_{m, m}$ is a matrix convergent to zero and $d(f(x), f(y)) \leq A d(x, y)$, $\forall x, y \in M)$, then we have the following result.

Theorem 2.3. Let $(M, d)$ is a complete $\mathbb{R}_{+}^{m}$-metric space, $f: M \rightarrow M$ be an A-contraction and $g: M \rightarrow M$ be an operator for which there exists $\eta=\left(\eta_{1}, \cdots, \eta_{m}\right) \in \mathbb{R}_{+}^{m}$ with $\eta_{i}>0$ for every $i \in\{1, \cdots, m\}$, such that $d(f(x), g(x)) \leq \eta$, for every $x \in M$. Denote by $x_{f}^{*}$ the unique fixed point of $f$. Then, we have that

$$
d\left(g^{n}(x), x_{f}^{*}\right) \leq\left(I_{m}-A\right)^{-1} \eta+A^{n}\left(I_{m}-A\right)^{-1} d(x, f(x)), \forall x \in M, \forall n \in \mathbb{N}^{*} .
$$

\section{Remark 2.1.}

Consider Problem I with $f: M \rightarrow M$ be a $\psi$-Picard operator. In this case, we have

$$
d\left(g^{n}(x), x_{f}^{*}\right) \leq \psi\left(d\left(g^{n}(x), f\left(g^{n}(x)\right)\right), \forall x \in M, \forall n \in \mathbb{N}^{*} .\right.
$$

By this relation, we obtain that, if $g$ is continuous and the sequence $\left(g^{n}(x)\right)_{n \in \mathbb{N}}$ is $f$-asymptotically regular (i.e., $d\left(g^{n}(x), f\left(g^{n}(x)\right)\right) \rightarrow 0$ as $n \rightarrow \infty$ for all $x \in M)$, then $g$ is a Picard operator with $x_{g}^{*}=x_{f}^{*}$.

\section{Results concerning Problem II.}

We start this section by the following result of Browder-Petryshyn (see [7]), given in terms of Picard operators.

Theorem 3.1. Let $B$ be a Banach space and $f: B \rightarrow B$ be a bounded linear operator. Suppose that $f$ is Picard. Let $g: B \rightarrow B$ be a constant operator, i.e., there exists $y \in B$ such that $g(x)=y$, for every $x \in B$. Then, for each $y \in\left(1_{B}-f\right)(B)$ the operator $f+g$ is Picard.

The main result of this section is the following. 
Theorem 3.2. Let $B$ be a Banach space and $f, g: B \rightarrow B$ be two operators. We suppose:

(1) $f$ is a strong contraction with respect to equivalent norms $\|\cdot\|_{\epsilon}$, where $0<\epsilon<1$;

(2) $g$ is an $l_{g}$-contraction with respect to each $\|\cdot\|_{\epsilon}$, for $0<\epsilon<1$.

Then, the operator $f+g$ is Picard.

Proof. Let $\epsilon>0$ such that $l_{g}+\varepsilon<1$. Then, the operator $f+g$ is a $\left(l_{g}+\varepsilon\right)$ contraction with respect to $\|\cdot\|_{\epsilon}$.

Example 3.1.

Let $(B,|\cdot|)$ be a Banach space and $T$ be given by Example 1.2. Since there exists $\left.l_{g} \in\right] 0,1[$ such that

$$
\left|g(t, u)-g(t, v) \leq l_{g}\right| u-v \mid, \forall t \in[a, b], \forall u, v \in B,
$$

we get that the operator $T$ is an $l_{g}$-contraction with respect to a Bielecki norm $\|\cdot\|_{\tau}$ on $C([a, b], B)$, for every $\tau>0$. Let $S$ be the operator given in Example 1.3. Then, $T+S$ is a Picard operator on $\left(C([a, b], B),\|\cdot\|_{\infty}\right)$. Indeed, $T+S$ is a $\left(l_{g}+\frac{l_{K}}{\tau}\right)$-contraction with respect to $\|\cdot\|_{\tau}$, where $\tau>0$ is such that $l_{g}+\frac{l_{K}}{\tau}<1$. Since the norms $\|\cdot\|_{\infty}$ and $\|\cdot\|_{\tau}$ are Lipschitz equivalent, the conclusion follows.

For other examples of this type see [32]. These examples suggest the following result.

Theorem 3.3. Let $(B,|\cdot|)$ be a Banach space and $\mathbb{B}:=\left(C([a, b], B),\|\cdot\|_{\tau}\right)$ be the Banach space of continuous abstract functions on $[a, b]$ with values in $B$ endowed with a Bielecki norm $\|\cdot\|_{\tau}$ corresponding to $\tau>0$. Let $T, S: \mathbb{B} \rightarrow \mathbb{B}$ be two operators. We suppose that:

(1) there exists $\left.l_{T} \in\right] 0,1[$ such that

$$
|T x(t)-T y(t)| \leq l_{T}|x(t)-y(t)|, \forall t \in[a, b], \forall x, y \in \mathbb{B}
$$

(2) there exists $c>0$ such that

$$
|S x(t)-S y(t)| \leq \frac{c}{\tau}\|x-y\|_{\tau} e^{\tau(t-a)}, \forall t \in[a, b], \forall x, y \in \mathbb{B} \text { and } \tau>0 .
$$

Then, the operators $T, S$ and $T+S$ are Picard in $\left(\left(C([a, b], B),\|\cdot\|_{\infty}\right)\right.$

Proof. We will prove that $T+S$ is a Picard operator. Indeed, for all $t \in[a, b]$, $x, y \in \mathbb{B}$ and $\tau>0$, we have

$$
|(T+S) x(t)-(T+S) y(t)| \leq l_{T}|x(t)-y(t)|+\frac{c}{\tau}\|x-y\|_{\tau} e^{\tau(t-a)} .
$$


Then

$$
\|(T+S) x-(T+S) y\|_{\tau} \leq\left(l_{T}+\frac{c}{\tau}\right)\|x-y\|_{\tau} .
$$

Since $l_{T}<1$, there exists $\tau>0$ such that $l_{T}+\frac{c}{\tau}<1$. This shows that $T+S$ is a contraction and hence a Picard operator.

\section{Example 3.2.}

Let $f, g: \mathbb{R} \rightarrow \mathbb{R}$ be two mappings defined by $f(x)=\frac{1}{3} x$ and $g(x)=-x$. In this case, $f$ is a Picard operator, $g$ is not a Picard operator, but $f+g: \mathbb{R} \rightarrow \mathbb{R}$ is a Picard operator.

By the above considerations, the following open question also arises.

Problem C. Let $B$ be a Banach space and $f: B \rightarrow B$ be an operator. In which conditions the operator $1_{B}-f$ is Picard ? Some basic references for this problem are [8], [9], [25], ...

\section{References}

[1] H. Amann, Ordinary Differential Equations, W. De Gruyter, 1990.

[2] V. Barbu, Nonlinear Semigroups and Differential Equations in Banach Spaces, Nordhooff, 1976.

[3] L. Berezansky, E. Bravermann, Preservation of the exponential stability under perturbations of linear delay impulsive differential equations, Zeitschrift Anal. Anwendungen 14 (1995), 157-174.

[4] V. Berinde, A. Petruşel, M.-A. Şerban, I.A. Rus, The retractiondisplacement condition in the theory of fixed point equation with a convergent iterative algorithm, in: Mathematical Analysis, Approximation Theory and Their Applications, Springer, 2016.

[5] S.R. Bernfeld, V. Lakshmikantham, An Introduction to Nonlinear Boundary Value Problems, Academic Press, 1974.

[6] P.T. Boggs, J.E. Dennis, A stability analysis for perturbed nonlinear iterative methods, Math. Comput. 30 (1976), 199-215.

[7] F.E. Browder, W.V. Petryshyn, The solution by iteration of linear functional equations in Banach spaces, Bull. Amer. Math. Soc. 72 (1966), 566-570.

[8] A. Buică, Coincidence Principles and Applications (in Romanian), Cluj University Press, 2001. 
Vol. LVI (2018) Stability of Picard operators under operator perturbations 11

[9] A. Buică, I.A. Rus, M.-A. Şerban, Zero point principle of ball-near identity operators and applications to implicit operator problem, Fixed Point Theory 21 (2020), no. 1 (to appear).

[10] T.A. Burton, Stability by fixed point methods for highly nonlinear delay equations, Filomat 5 (1) (2004), 3-20.

[11] T.A. Burton, Stability of Fixed Point Theory for Functional Differential Equations, Dover Publ., 2006.

[12] A. Granas, J. Dugundji, Fixed Point Theory, Springer, 2003.

[13] K. Goebel, On the spaces of strong contractions, Bull. Acad. Polon. Sci. 15 (5) (1967), 313-316.

[14] A. Halanay, Quelque questions de la théorie de la stabilité pour les sysstemes aux differences finies, Arch. Rational Mech. Anal. 12 (1963), 150-154.

[15] A. Halanay, Differential Equations: Stability, Oscillations, Time Lag, Academic Press, 1966.

[16] J. Hale, Theory of Functional Differential Equations, Springer, 1977.

[17] M.C. Irwin, Smooth Dynamical Systems, Academic Press, 1980.

[18] W.A. Kirk, B. Sims (eds.), Handbook of Metric Fixed Point Theory, Kluwer Acad. Publ., 2001.

[19] J.P. LaSalle, The Stability of Dynamical Systems, SIAM, 1976.

[20] B.S. Jovanović, S.V. Lemeshevsky, P.P. Matus, Stability of solutions of differential-operator and operator-difference equations with respect to perturbation of operators, Kragujevac J. Math. 30 (2007), 59-88.

[21] V. Lakshmikantham, S. Leela, Differential and Integral Inequalities, Academic Press, 1969.

[22] N. Lungu, I.A. Rus, On a functional Volterra-Fredholm integral equation via Picard operators, J. Math. Ineq. 3 (2009), 519 - 527.

[23] M. Megan, Sasu A.L., Sasu B., Asymptotic Behaviors for Evolution Equations Families, Mirton, 2003.

[24] V. Mureşan, Functional-Integral Equations, Mediamira, 2003.

[25] J. Ortega, W. Rheinboldt, Iterative Solutions of Nonlinear Equations in Several Variables, Academic Press, 1970.

[26] J. Peetre, I.A. Rus, Sur la positivité de la fonction de Green, Math. Scand. 21 (1967), 80-89. 
[27] S. Reich, A.J. Zaslavski, A stability result in fixed point theory, Fixed Point Theory 6, No. 1 (2005), 113-118.

[28] J.W. Robin, On Hadamard and Peron theorem, Prepint, 1999.

[29] I.A. Rus, Approximation of fixed points of generalized contraction mappings, in: Topics in Numerical Analysis II, Dublin, 1975, 157-161.

[30] I.A. Rus, Metrical Fixed Point Theorems, Babeş-Bolyai University Cluj-Napoca, 1979.

[31] I.A. Rus, Differential Equations, Integral Equations and Dynamical Systems (in Romanian), Transilvania Press Cluj-Napoca, 1996.

[32] I.A. Rus, A class of nonlinear functional-integral equations via Picard operators, Analele Univ. Craiova, Mat-Inform. 28 (2001), 10-15.

[33] I.A. Rus, Results and problems in Ulam stability of operatorial equations and inclusions, in: Handbook of Functional Equations: Stability Theory, Ed. T.M. Rassias, Springer, 2014, 323-352.

[34] I.A. Rus, Remarks on LaSalle conjecture on global asymptotic stability, Fixed Point Theory 17, No. 1 (2016), 159-172.

[35] I.A. Rus, Convergence results for fixed point iterative algorithms in metric spaces, Carpathian J. Math. 35, No. 2 (2019), 209-220.

[36] I.A. Rus, A. Petruşel, G. Petruşel, Fixed Point Theory, Cluj University Press, 2008.

[37] C. Vladimirescu, Existence of asymptotically stable solution to a nonlinear integral equation of mixed type, Fixed Point Theory 20, No. 1 (2019), 337-348.

Adrian Petruşel

Department of Mathematics, Babeş-Bolyai University

M. Kogălniceanu Street, no. 1

Cluj-Napoca, Romania

Academy of Romanian Scientists,

Bucharest, Romania

E-mail: petrusel@math.ubbcluj.ro

Ioan A. Rus

Department of Mathematics, Babeş-Bolyai University

M. Kogălniceanu Street, nr. 1

Cluj-Napoca, Romania

E-mail: iarus@math.ubbcluj.ro 